consecutive patients diagnosed by endometrial sampling as having an endometrioid grade $1 /$ grade 2 endometrial cancer. TVS and MRI were performed prior to surgical staging for assessing MI, which was estimated using subjective examiner's impression and Karlsson's method for both TVS and MRI. During surgery, intraoperative assessment of MI was also performed. Definitive pathological study considered as reference standard.

CUSUM graphs have been used to determine the number of necessary cases to reach the competence, admitting an error rate of between $10 \%$ and $25 \%$ as the expected precision of an expert is approximately $85 \%$.

Result(s)* The main sonographer of this study achieved proficiency after having sonographically evaluated 39 patients.

Conclusion* There is a learning process for the ultrasound assessment of the degree of myometrial infiltration that influences the diagnostic performance of transvaginal ultrasound. The learning curve of the lead sonographer in this study showed that she reached proficiency after sonographic study of 39 patients.

\section{FREQUENCY AND DURATION OF POST-MENOPAUSAL BLEEDING - RISK STRATIFICATION FOR ENDOMETRIAL CANCER}

L Honeyman, J Davies, S Kolhe, J Dasgupta, D Casayuran, A Phillips. Derby Gynaecological Cancer Centre, Derby, UK

\subsection{6/ijgc-2021-ESG0.193}

Introduction/Background* Recurrent bleeding can suggest a presence of cancer and has been incorporated into some risk prediction models for cancer in patients with PMB. However uniformed descriptions of PMB are not consistently used. We aimed to assess if the frequency or duration of bleeding allows identification of women at higher risk of cancer undergoing hysteroscopy for PMB

Methodology A retrospective review of hysteroscopy records from Royal Derby Hospital. Bleeding was defined in terms of number of episodes $(1,2,2+)$ and duration and was compared with histopathological records. Cases were identified between 2017 and 2019.

Result(s)* Between 2017 and 2019, 1101 women underwent a hysteroscopy for PMB. Seven were excluded as histology results were not obtained. Of the 1094 women included, 98 cancers were identified (9\%).

Regarding bleeding frequency; 184 women were excluded as there was insufficient data recorded. Of the remaining 910 women no significant difference was seen in the rate of cancer with different bleeding episodes; one episode (9.6\%), two episodes $(5.6 \%)$ or more than two episodes $(9 \%)$ $(\mathrm{p}>0.05)$.

Regarding character of bleeding; 556 were excluded as they did not have an explicit duration of bleeding recorded. Of the remaining $538 ; 409$ had a short ( $\leq 7$ days) duration, with $8(2 \%)$ cancers identified, 108 had an intermediate (1-4 weeks) duration with $12(11 \%)$ cancers identified, and of 21 with a long ( $>4$ weeks) duration of bleeding $4(19 \%)$ had a cancer identified. These results were highly significant ( $p$ $=<0.00001)$
In women who described unspecified cyclical/regular bleeding $(\mathrm{n}=98) 10$ cancers were detected $(10 \%)$

Conclusion* Accurately characterising bleeding duration is a more meaningful predictor of malignancy than episodes of bleeding. Standards should be developed to enable clinical history as well as radiological findings to triage care.

\section{3 AKR1C3 - A POTENTIAL PROGNOSTIC BIOMARKER FOR PATIENTS WITH ENDOMETRIAL CARCINOMAS}

${ }^{1} \mathrm{M}$ Hojnik*, ${ }^{2 ; 3} \mathrm{~N}$ Kenda Šuster, ${ }^{2 ; 3 \zeta ̌ ~ S m r k o l j, ~}{ }^{4} S$ Frković Grazio, ${ }^{2}$ Verdenik, ${ }^{1} \mathrm{~T}$ Lanišnik Rižner. 'Institute of Biochemistry, Faculty of Medicine, University of Ljubljana, 1000 Ljubljana, Slovenia; ${ }^{2}$ Division of Gynecology, Department of Obstetrics and Gynecology, University Medical Centre Ljubljana, 1000 Ljubljana, Slovenia; ${ }^{3}$ Chair for Gynecology and Obstetrics, Medical Faculty, University of Ljubljana, Slovenia; ${ }^{4}$ Division of Gynecology, Department of Pathology, University Medical Centre Ljubljana, 1000 Ljubljana, Slovenia

\subsection{6/ijgc-2021-ESG0.194}

Introduction/Background* Endometrial cancer is the most common gynecological cancer diagnosis in developed countries. Personalized treatments for these cancers depend on identification of prognostic and predictive biomarkers that allow stratification of patients.

The aldo-keto reductase (AKR) superfamily is getting attention in cancer research, because of AKR's involvements in important biochemical processes. The enzyme AKR1C3 has many functions, which include production of prostaglandins, androgens, estrogens, and metabolism of different chemotherapeutics. AKR1C3 is thus implicated in the pathophysiology of different cancers.

Methodology In our study, we evaluated the immunohistochemical (IHC) staining of AKR1C3 in 123 paraffin-embedded samples of endometrial cancer and statistically examined possible correlations between expression of AKR1C3 and clinicopathological data (survival, stage of disease, lymphovascular invasion, menopausal status, parous status, smoking, use of hormone replacement therapy).

Result(s)* In endometrioid endometrial carcinoma, high AKR1C3 IHC expression correlated with better overall survival $(\mathrm{p}=0.008)$ and with disease-free survival $(\mathrm{p}=0.027)$. Significantly higher percentages of positive epithelial cells were seen for adjacent nonneoplastic endometrium compared to endometrioid endometrial cancer $(\mathrm{p}=1 \times 10-4)$.

Conclusion* These results demonstrate the potential use of AKR1C3 as an independent prognostic biomarker in endometrioid endometrial cancer. Clinically, this might mean that levels of AKR1C3 in endometrioid endometrial cancer could be determined preoperatively on diagnostic tissue samples to allow prediction of the cancer behavior and stratification of the patients for more personalized treatments.

\section{Disclosures}

Conflicts of Interest The authors declare no conflict of interest. Study was supported by the grants J3-2535 to T.L.R. from the Slovenian Research Agency.

Ethical Issues The study was approved by the National Medical Ethics Committee of the Republic of Slovenia (0120-701/ 2017-6). 